\title{
REFERENCES
}

1. J. D. Buckholtz, Concerning an approximation of Copson, Proc. Amer. Math. Soc. 14 (1963), 564-568.

2. E. T. Copson, An approximation connected with $e^{-3}$, Proc. Edinburgh Math. Soc. (2) 3 (1932/1933), 201-206.

3. G. Polya and G. Szegö, Aufgaben und Lehrsätze aus der Analysis. I, Springer, Berlin, 1925.

4. S. Ramanujan, Collected papers, Cambridge Univ. Press, Cambridge, 1927.

5. J. Riordan, An introduction to combinatorial analysis, Wiley, New York, 1958.

DUKE UNIVERSITY

\section{ON CLASSES OF UNIVALENT CONTINUED FRACTIONS}

T. L. HAYDEN ${ }^{1}$ AND E. P. MERKES ${ }^{2}$

1. Introduction. From results of Leighton and Scott [3], there is a unique one-to-one correspondence between formal power series $w^{-1}+\sum_{n=2}^{\infty} c_{n} w^{-n}$ and $C$-fractions

$$
F(w)=\frac{1}{w}-\frac{a_{1}}{w^{\delta_{1}}}-\frac{a_{2}}{w^{\delta_{2}}}-\cdots-\frac{a_{n}}{w^{\delta_{n}}}-\cdots,
$$

where $\delta_{n}$ is an integer, $\delta_{1} \geqq 0, \delta_{n+1}+\delta_{n} \geqq 1$, and $a_{n+p}=0$ whenever $a_{p}=0$ for $n=1,2, \ldots$. For a fixed continued fraction (1.1), let $K_{F}$ denote the class of formal power series which correspond to $C$-fractions of the form

$$
\frac{1}{w}-\frac{a_{1}^{\prime}}{w^{\delta_{1}}}-\frac{a_{2}^{\prime}}{w^{\delta_{2}}}-\cdots-\frac{a_{n}{ }^{\prime}}{w^{\delta_{n}}}-\cdots,
$$

where $\left|a_{n}^{\prime}\right| \leqq\left|a_{n}\right|, n=1,2, \cdots$. In order that each power series in $K_{F}$ represent an analytic function in $|w| \geqq 1$ it is necessary and sufficient that $\left|a_{n}\right| \leqq g_{n}\left(1-g_{n-1}\right)$, where $0<g_{n-1} \leqq 1, n=1,2, \cdots$, and $g_{p-1}=1$ if and only if $a_{p}=0[2$, p. 374]. Conditions on the parameters $g_{n}$ of the chain sequence $\left\{g_{n}\left(1-g_{n-1}\right)\right\}_{n=1}^{\infty}$ which imply that each

Received by the editors October 16, 1963.

${ }^{1}$ Sponsored by the Mathematics Research Center, U. S. Army, Madison, Wisconsin under Contract No. DA-11-022-ORD-2059. 然

2 Sponsored in part by the Mathematics Research Center, U. S. Army, Madison, Wisconsin under Contract No. DA-11-022-ORD-2059 and in part by the Air Force Office of Scientific Research. 
function in $K_{F}$ is univalent in $|w|>1$ are determined in $\S 2$. A similar problem for a class of analytic functions with certain $J$-fraction expansions is also considered. In $\$ 3$ conditions on the parameters which imply that each function in $K_{F}$ is starlike with respect to the origin in $|w|>1$ are given. These results extend those of Thale [7], Perron [6], Merkes and Scott [5] which, in our terminology, treat the class $K_{F}$ where $F$ is given by (1.1) with $a_{n}=a \neq 0, \delta_{2 n-1}=\delta$, $\delta_{2 n}=1, \delta$ a nonnegative integer, $n=1,2, \cdots$.

2. Univalence. Let $F(w)$ be a fixed $C$-fraction (1.1) and let $K_{F}$ be the associated class of formal power series.

THEOREM 2.1. Each $f(w) \in K_{F}$ is analytic and univalent in $|w|>1$ if $\left\{\left|a_{j}\right|\right\}_{j=1}^{\infty}$ is a chain sequence with parameters $g_{j}, 0<g_{j} \leqq 1, j=0$, $1,2, \cdots$, and

$$
\Lambda=\sum_{j=1}^{\infty}\left|\delta_{j}\right| \prod_{p=1}^{j} \frac{1-g_{p-1}}{g_{p}} \leqq 1 .
$$

Furthermore, if $\delta_{j} \geqq 0, j=1,2, \cdots$, and $\left\{g_{j}\right\}$ is the maximal parameter sequence $[8, p .81]$, there is a function in $K_{F}$ which is not univalent in $|w|>R$ for any $R<1$ whenever $\Lambda=1$.

PROOF. For each nonnegative integer $n$, define

$$
f_{n}(w)=\frac{1}{w^{\delta_{n}}}-\frac{a_{n+1}^{\prime}}{w^{\delta_{n+1}}}-\frac{a_{n+2}^{\prime}}{w^{\delta_{n+3}}}-\cdots,
$$

where $\delta_{0}=1$ and $f_{0}(w) \in K_{F}$. Since $\left|a_{j}^{\prime}\right| \leqq\left|a_{j}\right|$, where $\left\{\left|a_{j}\right|\right\}$ in (1.1) is a chain sequence, each $f_{n}(w)$ is analytic in $|w| \geqq 1[2]$ and

$$
0<\left|f_{n}(w) w^{\delta_{n}}\right| \leqq 1 / g_{n}
$$

for $|w| \geqq 1\left[8\right.$, p. 46]. For a fixed $w_{1}$ in $|w| \geqq 1$, it is easily shown by (2.2) that

$$
f_{0}(w)-f_{0}\left(w_{1}\right)=-\sum_{j=0}^{m}\left(w^{\delta_{j}}-w_{1}^{\delta_{j}}\right) \prod_{p=0}^{j} a_{p}^{\prime} f_{p}(w) f_{p}\left(w_{1}\right)+R_{m},
$$

where $a_{0}^{\prime}=1=\delta_{0}$ and

$$
R_{m}=a_{m+1}^{\prime}\left[f_{m+1}(w)-f_{m+1}\left(w_{1}\right)\right] \prod_{p=0}^{m} a_{p}^{\prime} f_{p}\left(w_{1}\right) f_{p}(w) .
$$

By (2.3) and the fact that $\left|a_{j}^{\prime}\right| \leqq g_{j}\left(1-g_{j-1}\right), j=1,2, \cdots$, we obtain, for $|w| \geqq 1$, 


$$
\begin{aligned}
\left|R_{m}\right| & \leqq\left[|w|^{-k_{m}+1}\left|w_{1}\right|^{-k_{m}}+|w|^{-k_{m}}\left|w_{1}\right|^{-k_{m+1}}\right] \frac{g_{m+1}}{g_{0}^{2}} \prod_{p=1}^{m+1} \frac{1-g_{p-1}}{g_{p}} \\
& \leqq \frac{2}{g_{0}^{2}} \prod_{p=1}^{m+1} \frac{1-g_{p-1}}{g_{p}},
\end{aligned}
$$

where $k_{n}=\delta_{0}+\delta_{1}+\cdots+\delta_{n}>0$ by the conditions $\delta_{0}=1, \delta_{n-1}+\delta_{n} \geqq 1$, $n=1,2, \cdots$. The convergence of the series $\Lambda$ in (2.1) now implies $R_{m} \rightarrow 0$ uniformly as $m \rightarrow \infty$ for $|w| \geqq 1$. Hence, by (2.4),

$$
f_{0}(w)-f_{0}\left(w_{1}\right)=-\sum_{j=0}^{\infty}\left(w^{\delta_{j}}-w_{1}^{\delta_{j}}\right) \prod_{p=0}^{j} a_{p}^{\prime} f_{p}(w) f_{p}\left(w_{1}\right)
$$

and

$$
f_{0}^{\prime}(w)=-\sum_{j=0}^{\infty} \delta_{j} w^{\delta_{j-1}} \prod_{p=0}^{j} a_{p}^{\prime} f_{p}^{2}(w), \quad|w| \geqq 1 .
$$

Now for $|w| \geqq 1,\left|w_{1}\right| \geqq 1$,

$$
\left|w^{-k_{j-1}} w_{1}^{-k_{j}}-w^{-k_{j}} w_{1}^{-k_{j-1}}\right| \leqq\left|w-w_{1}\right|\left|\delta_{j}\right|,
$$

where $k_{j}=\delta_{0}+\cdots+\delta_{j}>0, j=0,1,2, \cdots$. In conjunction with (2.3), this shows by (2.5) that

$$
\left|\frac{f_{0}(w)-f_{0}\left(w_{1}\right)}{\left(w-w_{1}\right) f_{0}(w) f_{0}\left(w_{1}\right)}+1\right| \leqq \Lambda,
$$

where $\Lambda$, given by (2.1), does not exceed unity. Since the function within the absolute-value symbol, when appropriately defined at $w=w_{1}$, is analytic in $|w| \geqq 1$, the maximum modulus principle gives strict inequality for $|w|>1$ provided $f_{0}(w) \not w^{-1}$. In particular, $f_{0}(w) \neq f_{0}\left(w_{1}\right)$ for $w \neq w_{1},|w|>1,\left|w_{1}\right|>1$. This proves the first part of the theorem.

Let

$$
F_{0}(w)=\frac{1}{w}+\frac{\left|a_{1}\right|}{w^{\delta_{1}}}-\frac{\left|a_{2}\right|}{w^{\delta_{2}}}-\cdots,
$$

which is in $K_{F}$, and let

$$
F_{j}(w)=\frac{1}{w^{\delta_{j}}}-\frac{\left|a_{j+1}\right|}{w^{\delta_{j+1}}}-\cdots, \quad j=1,2, \cdots .
$$

When $\left\{g_{n}\right\}$ is the maximal parameter sequence of $\left\{\left|a_{n}\right|\right\}, F_{j}(1)$ $=1 / g_{j}, j=1,2, \cdots\left[8\right.$, p. 81]. Since $\left|a_{j}\right|=g_{j}\left(1-g_{j-1}\right), j=1,2, \cdots$, 
it follows from (2.6) that, for $\delta_{j} \geqq 0$,

$$
\frac{F_{0}^{\prime}(1)}{\left[F_{0}(1)\right]^{2}}=-1+\sum_{j=1}^{\infty} \delta_{j} \prod_{p=1}^{j} \frac{1-g_{p-1}}{g_{p}}=-1+\Lambda \text {. }
$$

Therefore, if $\Lambda=1, F_{0}(w)$ is not univalent in $|w|>R$ for any $R<1$.

If a formal power series $f(w)$ corresponds to a $J$-fraction, it is of ten possible to obtain a larger region of analyticity and univalence for $f(w)$ than that obtained from Theorem 2.1. A result of this kind is given by the following:

TheOREM 2.2. Let $b_{n}$ and $\beta_{n}>0$ be complex numbers, $n=1,2, \cdots$. Let $D$ be a region such that $w \in D$ implies $\left|w-b_{n}\right|>\beta_{n}, n=1,2, \cdots$. The J-fraction

$$
\frac{1}{w-b_{1}}-\frac{a_{1}^{2}}{w-b_{2}}-\cdots-\frac{a_{n}^{2}}{w-b_{n+1}}-\cdots
$$

represents an analytic univalent function in $D$ provided $\left\{\left|a_{n}^{2}\right| / \beta_{n} \beta_{n-1}\right\}_{n=1}^{\infty}$ is a chain sequence with parameters $g_{n}, 0<g_{n} \leqq 1, n=0,1, \cdots$, such that

$$
L=\sum_{n=1}^{\infty} \frac{\beta_{1}}{\beta_{n+1}} \prod_{p=1}^{n} \frac{1-g_{p-1}}{g_{p}} \leqq 1 .
$$

The proof is similar to that of Theorem 2.1 and therefore is omitted. In particular, when $\left|a_{n}\right| \leqq N / 3, \beta_{n} \geqq \beta_{1}>0, n=1,2, \cdots$, the choice $g_{n}=2 / 3, n=0,1, \cdots$, shows $(2.8)$ is analytic and univalent in any region contained in the common part of the regions $\left|w-b_{n}\right|>\sqrt{ }(2) N / 2$. This statement includes Thale's results on $J$-fractions [7]. Indeed, if $\left|b_{n}\right| \leqq M / 3, n=1,2, \cdots$, then a domain of univalence of $(2.8)$ is $|w|>(3 \sqrt{ }(2) N+2 M) / 6$. Moreover, if Im $b_{n} \leqq 0, n=1,2, \cdots$, which is the case whenever $(2.8)$ is positive definite, then $\operatorname{Im} w>\sqrt{ }(2) N / 2$ is a domain of univalence of the $J$ fraction. Each of these results is sharp. For, by a suitable choice of the real number $a$, the function

$$
\begin{aligned}
& \frac{2}{3(w-a)-\sqrt{ }\left((w-a)^{2}+4 N^{2} / 9\right)} \\
& =\frac{1}{w-a}-\frac{N^{2} / 9}{w-a}+\frac{N^{2} / 9}{w-a}+\frac{N^{2} / 9}{w-a}+\cdots,
\end{aligned}
$$

whose derivative vanishes at $w=a+\sqrt{ }(2) N i / 2$, is not univalent in a given open region which properly contains one of these domains of univalence. 
3. Starlikeness. As a simple consequence of (2.7) we have the following sufficient condition for each power series in $K_{F}$ to represent a starlike function.

THEOREM 3.1. Each $f(w) \in K_{F}$ is analytic, univalent, and starlike with respect to the origin in $|w|>1$ if $\left\{\left|a_{j}\right|\right\}_{j=1}^{\infty}$ is a chain sequence with parameters $g_{j}, 0<g_{j} \leqq 1$, and

$$
\Lambda \leqq\left[g_{0}\left(2-g_{0}\right)\right]^{1 / 2},
$$

where $\Lambda$ is defined in (2.1).

Proof. Let $f(w) \in K_{F}$ correspond to the $C$-fraction (1.2). From (2.7), we obtain

$$
\left|\frac{w f^{\prime}(w)}{f(w)}+w f(w)\right| \leqq|w f(w)| \Lambda .
$$

This implies $\operatorname{Re}\left\{w f^{\prime}(w) / f(w)\right\} \leqq 0$ for $|w|>1$, which is sufficient for starlikeness with respect to the origin, provided

$$
\cos \theta \geqq \Lambda, \quad \theta=\arg \{w f(w)\} .
$$

Since $[8$, p. 46$]$

$$
\left|w f(w)-\frac{1}{g_{0}\left(2-g_{0}\right)}\right| \leqq \frac{1-g_{0}}{g_{0}\left(2-g_{0}\right)}, \quad|w|>1,
$$

it follows that

$$
\cos \theta \geqq\left[g_{0}\left(2-g_{0}\right)\right]^{1 / 2} .
$$

In conjunction with (3.1) this establishes the theorem.

In general, it is conjectured that, for any $C$-fraction (1.1) such that the hypothesis of Theorem 2.1 hold, each $f(w) \in K_{F}$ is analytic, univalent, and starlike with respect to the origin in $|w|>1$.

Let, in particular, $F(w)$ be the $C$-fraction (1.1) with $a_{n}=g_{0}\left(1-g_{0}\right)$, $1 / 2 \leqq g_{0}<1, \delta_{2 n-1}=0, \delta_{2 n}=1, n=1,2, \cdots$. By Theorem 3.1, each $f(w) \in K_{F}$ is starlike with respect to the origin if $g_{0}>.60$. This is an improvement over the previously obtained [5] lower estimate of $(3-\sqrt{ }(3)) / 2$. Recently, F. V. Atkinson [1] has verified a conjecture of the authors that the radius of starlikeness is the radius of univalence in this case and in the slightly more general situation treated in [5].

An argument similar to that of Theorem 3.1 gives the following result on starlikeness of $J$-fractions. 
THEOREM 3.2. Let $b_{n}$ and $\beta_{n}>0, n=1,2, \cdots$, be complex numbers such that $\left|w-b_{n}\right|>\beta_{n}, n=1,2, \cdots$, whenever $|w|>R$. The J-fraction (5.8) is analytic, univalent, and starlike with respect to the origin in $|w|>R$ provided $b_{1}=0$ and $\left\{\left|a_{n}\right|^{2} / \beta_{n} \beta_{n+1}\right\}_{n=1}^{\infty}$ is a chain sequence with parameters $g_{n}, 0<g_{n} \leqq 1$, and

$$
\left[g_{0}\left(2-g_{0}\right)\right]^{1 / 2} \geqq L
$$

where $L$ is given by (2.9).

A similar result for the case $b_{1} \neq 0$ can be deduced but because of its complexity is omitted. This result slightly improves those in [4] and lends credence to the conjecture that the radius of starlikeness is the same as the radius of univalence for bounded $J$-fractions.

\section{REFERENCES}

1. F. V. Atkinson, A value region problem occurring in the theory of continued fractions, Technical Report, Mathematics Research Center, University of Wisconsin, Madison, Wis., 1963.

2. R. E. Lane and H. S. Wall, Continued fractions with absolutely convergent even and odd parts, Trans. Amer. Math. Soc. 67 (1949), 366-380.

3. W. Leighton and W. T. Scott, A general continued fraction expansion, Bull. Amer. Math. Soc. 45 (1939), 596-605.

4. E. P. Merkes, Bounded J-fractions and univalence, Michigan Math. J. 6 (1959), 395-400.

5. E. P. Merkes and W. T. Scott, On univalence of a continued fraction, Pacific J. Math. 10 (1960), 1361-1369.

6. O. Perron, Über ein Schlichtheitsschranke von James S. Thale, Bayer. Akad. Wiss. Math.-Natur. K1. S.-B. 1956 (1957), 233-236.

7. J. S. Thale, Univalence of continued fractions and Stieltjes transforms, Proc. Amer. Math. Soc. 7 (1956), 232-244. 1948.

8. H. S. Wall, Analytic theory of continued fractions, Van Nostrand, New York,

UNIVERSITY OF WISCONSIN 